\title{
Lactobacillus similis sp. nov., isolated from fermented cane molasses
}

Correspondence

Maki Kitahara

kitahara@jcm.riken.jp

\author{
Maki Kitahara, Mitsuo Sakamoto and Yoshimi Benno
}

\begin{abstract}
Microbe Division/Japan Collection of Microorganisms, RIKEN BioResource Center, Wako, Saitama 351-0198, Japan
\end{abstract}

\begin{abstract}
The taxonomic position of strain JCM $2765^{\top}$ isolated from fermented cane molasses in Thailand was reinvestigated. Strain JCM $2765^{\top}$ was originally identified as representing Lactobacillus buchneri on the basis of biochemical and physiological characteristics. In the present study, 16S rRNA gene sequence analysis of strain JCM $2765^{\top}$ demonstrated a low level of similarity with the type strain of L. buchneri (92.5\%) and high levels with those of Lactobacillus collinoides (97.6\%) and Lactobacillus paracollinoides (98.0\%). Ribotyping was applied to investigate the relationships between strain JCM $2765^{\top}, L$. collinoides and $L$. paracollinoides. The dendrogram based on ribotyping patterns showed one cluster for six strains of $L$. paracollinoides, and that strain JCM $2765^{\top}$ and L. collinoides JCM $1123^{\top}$ were each independent. Based on additional phenotypic findings and DNA-DNA hybridization results, strain JCM $2765^{\top}$ is considered to represent a novel species of the genus Lactobacillus, for which the name Lactobacillus similis sp. nov. is proposed. The type strain is JCM $2765^{\top}$ (=LMG $\left.23904^{\top}\right)$.
\end{abstract}

Strain JCM $2765^{\mathrm{T}}$ was one of 89 isolates from fermented cane molasses at alcohol plants in Thailand and was first described by Seki et al. (1989). Strain JCM $2765^{\mathrm{T}}$ was a facultatively anaerobic, non-spore-forming, Gram-positive rod-shaped bacterium, and produced lactic acid from glucose as a major product. It was identified by Seki et al. (1989) as representing Lactobacillus buchneri on the basis of biochemical and physiological characteristics compared with L. buchneri JCM $1115^{\mathrm{T}}$. In the present study, further genomic research revealed that strain JCM $2765^{\mathrm{T}}$ represents a novel species of the genus Lactobacillus.

The following eight strains were investigated: JCM $2765^{\mathrm{T}}$, Lactobacillus collinoides JCM $1123^{\mathrm{T}}$, and Lactobacillus paracollinoides JCM $11969^{\mathrm{T}}\left(=\mathrm{LA} 2^{\mathrm{T}}\right)$, JCM 1113, JCM 15728 (=LA3), JCM 15729 (=LA4), JCM 15730 (=LA7) and JCM 15731 (=LA8). The above L. paracollinoides strains were isolated from brewery environment and were described by Suzuki et al. (2004a, b, c, 2006). Strain JCM $2765^{\mathrm{T}}$ and L. collinoides JCM $1123^{\mathrm{T}}$ were grown on MRS (Merck) agar plates at 37 and $26{ }^{\circ} \mathrm{C}$, respectively, under

The GenBank/EMBL/DDBJ accession numbers for the $16 \mathrm{~S}$ rRNA gene sequences of strains JCM 2765 ${ }^{\top}, \mathrm{JCM} 11969^{\top}$, JCM 1113, JCM 15728, JCM 15729, JCM 15730 and JCM 15731 are AB282889 and AB473623-AB473628, respectively.

Minimum-evolution and maximum-parsimony phylogenetic trees based on 16S rRNA gene sequences showing the relationship between strain $\mathrm{JCM} 2765^{\top}$ and related species, table giving differential characteristics between strain JCM $2765^{\top}$, Lactobacillus collinoides and Lactobacillus paracollinoide, and table giving levels of DNA-DNA relatedness between strain $J C M 2765^{\top}$ and strains of $L$. collinoides and $L$. paracollinoides are available with the online version of this paper. anaerobic conditions. L. paracollinoides strains were grown in beer medium agar plates at $26{ }^{\circ} \mathrm{C}$ under anaerobic conditions. Beer medium was prepared as MRS medium dissolved with normally hopped beer instead of distilled water.

Physiological, biochemical and enzyme activity tests were performed by inoculation of API $50 \mathrm{CH}$ and API ZYM (bioMérieux) strips in triplicate according to the manufacturer's instructions. Production of $\mathrm{CO}_{2}$ from glucose fermentation, catalase activity, determination of $\mathrm{D}$ - and $\mathrm{L}-$ lactic acid production, and aesculin hydrolysis were reported by Seki et al. (1989). Approximately 1500 bases of the 16S rRNA gene sequences of strains JCM $2765^{\mathrm{T}}$, JCM $11969^{\mathrm{T}}$, JCM 1113 and JCM 15728-15731 were amplified by PCR with universal primers $27 \mathrm{~F}$ ( $5^{\prime}$-dAGAGTTTGATCCTGGCTCAG-3') and 1492R (5'-dGGTTACCTTGTTACGACTT-3') by using a Biometra Thermocycler Tgradient. PCR products were purified by using an Ultraclean PCR Clean-up kit (Mo Bio) and were sequenced by using a BigDye Terminator cycle sequencing kit (Applied Biosystems) and ABI PRISM 3100 Genetic Analyzer (Applied Biosystems). The closest recognized relatives of the isolates were determined by performing database searches, and sequences of closely related species were retrieved from the DDBJ, EMBL and GenBank nucleotide sequence databases. Phylogenetic analysis was performed with the CLUSTAL $X$ (version 1.83) program (Thompson et al., 1997) and a phylogenetic tree was constructed according to the neighbour-joining method (Saitou \& Nei, 1987). The topology of the tree was evaluated by bootstrap analysis of 1000 replicates by using 
the ClUSTAL X software. Minimum-evolution and maximum-parsimony phylogenetic trees were inferred by using the software package MEGA, version 4.0 (Tamura et al., 2007), according to the Kimura two-parameter model (Kimura, 1980). A species-specific PCR assay to identify $L$. paracollinoides was performed according to Suzuki et al. (2004b). The automated ribotyping device RiboPrinter microbial characterization system (Qualicon) was used for ribotyping in accordance with the manufacturer's instructions, with $E c o$ RI as a restriction enzyme. Ribopatterns were analysed by BioNumerics version 2.5 software (Applied Maths) and were compared by Pearson similarity coefficient analysis and the unweighted pair group method using arithmetic average (UPGMA) algorithm. For DNA-DNA hybridization experiments, bacterial DNA of seven strains, JCM $2765^{\mathrm{T}}$, L. collinoides JCM $1123^{\mathrm{T}}$, and L. paracollinoides JCM $11969^{\mathrm{T}}$, JCM 1113, JCM 15728, JCM 15729 and JCM 15730, was extracted from cells harvested from MRS broth (Difco) after $12 \mathrm{~h}$ as described by Kitahara et al. (2001) and was purified according to the methods of Saito \& Miura (1963). Levels of DNA-DNA relatedness were determined according to the method of Ezaki et al. (1989) by using photobiotin and microplates. Hybridization was performed at $45^{\circ} \mathrm{C}$ for $18 \mathrm{~h}$. The DNA G $+\mathrm{C}$ content was determined as described by Kitahara et al. (2001).
Approximately 1500 bases of the $16 \mathrm{~S}$ rRNA gene sequence of strain JCM $2765^{\mathrm{T}}$ were determined. The neighbourjoining, minimum-evolution and maximum-parsimony phylogenetic trees clearly indicated that strain JCM $2765^{\mathrm{T}}$ was related to strains in the genus Lactobacillus, as shown in Fig. 1 and Supplementary Figs S1 and S2 available in IJSEM Online. The phylogenetic trees showed clearly that strain JCM $2765^{\mathrm{T}}$ had a distinct line of descent with high bootstrap support. Strain JCM $2765^{\mathrm{T}}$ showed highest $16 \mathrm{~S}$ rRNA gene sequence similarity to L. collinoides JCM $1123^{\mathrm{T}}$ $(97.6 \%)$ and L. paracollinoides JCM $11969^{\mathrm{T}}$ (98.0\%). Strain JCM $2765^{\mathrm{T}}$ was originally identified by Seki et al. (1989) as representing L. buchneri based on biochemical and physiological characteristics, but the two taxa were not closely related in the present phylogenetic trees; 16S rRNA gene sequence similarity between strain JCM $2765^{\mathrm{T}}$ and the type strain of $L$. buchneri was $92.5 \%$. In the present study, $16 \mathrm{~S}$ rRNA gene sequences for six strains of $L$. paracollinoides were redetermined, and their sequence similarities were over $99.9 \%$. Strains JCM $11969^{\mathrm{T}}$, JCM 15728, JCM 15729 and JCM 15731 had identical sequences. The level of $16 \mathrm{~S}$ rRNA gene sequence similarity between $L$. paracollinoides JCM $11969^{\mathrm{T}}$ and L. collinoides JCM $1123^{\mathrm{T}}$ was $99.6 \%$ in the present study. However, L. paracollinoides can be differentiated from L. collinoides on the basis of

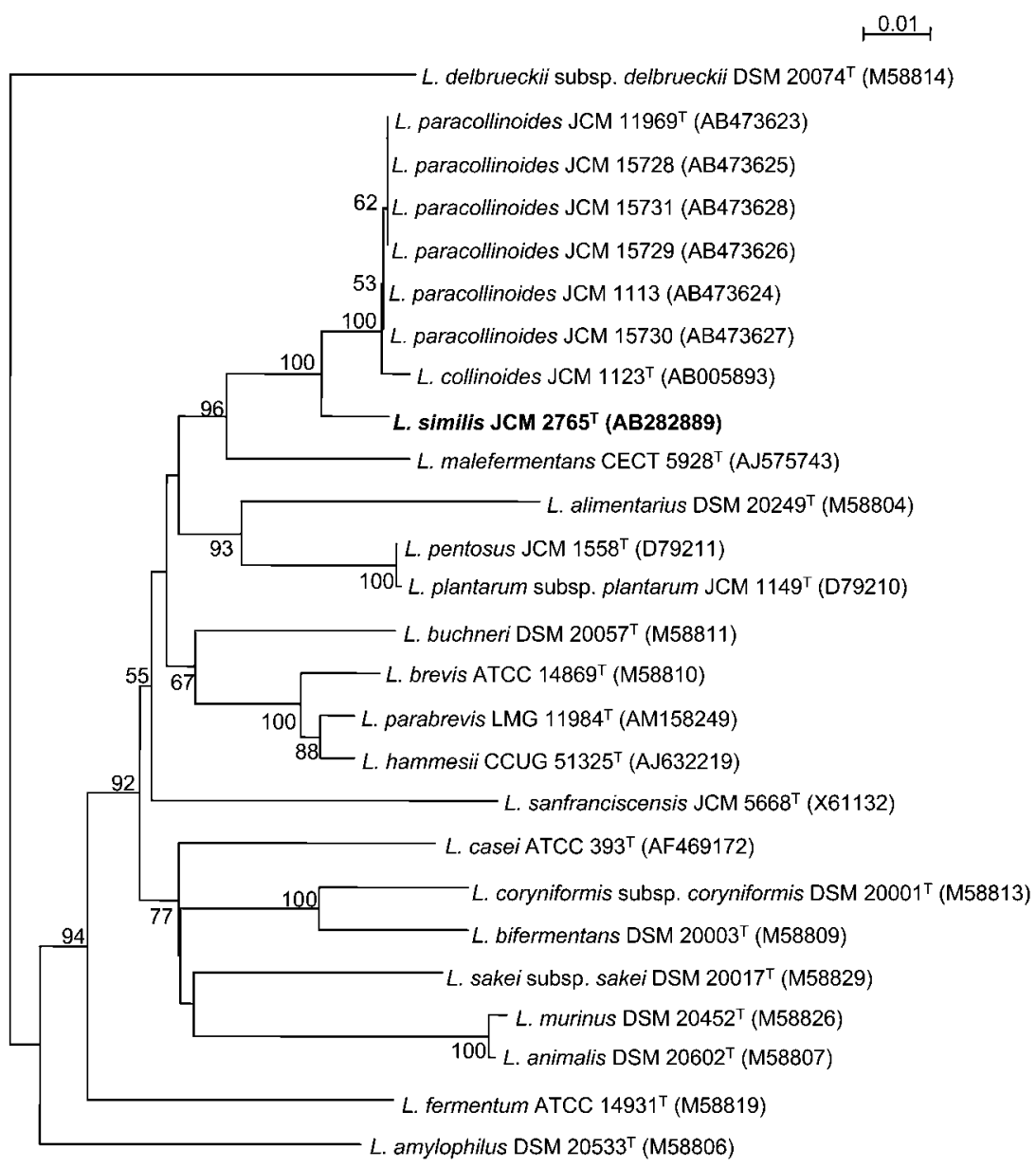

Fig. 1. Phylogenetic tree showing the relationship between strain JCM $2765^{\top}$ and representatives of related species. The tree was constructed by the neighbour-joining method based on 16S rRNA gene sequences. Numbers at nodes indicate percentage bootstrap values of 1000 replicates; only values $>50 \%$ are given. Accession numbers for $16 \mathrm{~S}$ rRNA gene sequences are given in parentheses for each strain. Bar, 0.01 substitutions per nucleotide position. Minimumevolution and maximum-parsimony trees are available as Supplementary Figs $\mathrm{S} 1$ and $\mathrm{S} 2$ in IJSEM Online. 
DNA-DNA relatedness and beer-spoiling ability (Suzuki et al., 2004a). Furthermore, species-specific primers based on intergenic spacer region sequences were published for use in the differentiation of beer-spoiling $L$. paracollinoides from non-beer-spoiling L. collinoides strains (Ehrmann \& Vogel, 2005; Suzuki et al., 2004b). In the present study, strain JCM $2765^{\mathrm{T}}$ had the same amplified band pattern as L. paracollinoides strains based on the specific primers for L. paracollinoides (data not shown), although L. collinoides JCM $1123^{\mathrm{T}}$ had a different amplified band pattern as reported by Ehrmann \& Vogel (2005). The specific primer for $L$. paracollinoides was not effective for differentiation between strain JCM $2765^{\mathrm{T}}$ and $L$. paracollinoides.

Ribotyping via the RiboPrinter system was applied to investigate the relationships between strain JCM $2765^{\mathrm{T}}, L$. collinoides and L. paracollinoides (Fig. 2). The dendrogram by ribotyping patterns showed one cluster for six strains of L. paracollinoides, and that strain JCM $2765^{\mathrm{T}}$ and $L$. collinoides JCM $1123^{\mathrm{T}}$ were each independent. Moreover, the ribotyping also showed that the six strains of $L$. paracollinoides fell into two distinct clusters as in the phylogenetic tree based on 16S rRNA gene sequences (Fig. 1). Four strains of L. paracollinoides, JCM $11969^{\mathrm{T}}$, JCM 15728, JCM 15729 and JCM 15731, had similar ribotyping patterns, consistent with levels of 16S rRNA gene sequence similarity.

Physiological and biochemical properties of strain JCM $2765^{\mathrm{T}}$ were determined via the API $50 \mathrm{CH}$ and API ZYM systems. The results are given in the species description below. Identical results were shown for strain JCM $2765^{\mathrm{T}}$ and L. collinoides JCM $1123^{\mathrm{T}}$ with the API ZYM system, but contrasting results were shown by the API $50 \mathrm{CH}$ system among strain JCM $2765^{\mathrm{T}}$, L. collinoides JCM $1123^{\mathrm{T}}$ and $L$. paracollinoides strains. The combination of carbohydrate utilization can be used to differentiate among the three taxa. For example, strain JCM $2765^{\mathrm{T}}$ showed positive and negative reactions for utilization of galactose and D-fructose, respectively, whereas those for L. collinoides JCM $1123^{\mathrm{T}}$ were both positive, and those for $L$. paracollinoides were both negative (Supplementary Table S1, available in IJSEM Online).
DNA-DNA hybridization experiments were performed among strain JCM $2765^{\mathrm{T}}$, L. collinoides JCM $1123^{\mathrm{T}}$ and $L$. paracollinoides JCM $11969^{\mathrm{T}}$, JCM 1113, JCM 15728, JCM 15729 and JCM 25730 (Supplementary Table S2, available in IJSEM Online). Low levels of DNA-DNA hybridization were found between strain JCM $2765^{\mathrm{T}}$ and L. collinoides JCM $1123^{\mathrm{T}}(6 \%)$, and also between strain JCM $2765^{\mathrm{T}}$ and L. paracollinoides strains $(7-16 \%)$. These low values indicated clearly that strain JCM $2765^{\mathrm{T}}$ belongs to a species distinct from $L$. collinoides and $L$. paracollinoides. Suzuki et al. (2004a) reported that levels of DNA-DNA relatedness values between $L$. collinoides JCM $1123^{\mathrm{T}}$ and $L$. paracollinoides strains were relatively low (46.8-57.6\%), but values in the present study were much lower (22$31 \%)$. These differences might be related to the hybridization temperature, but no information about hybridization temperature was reported by Suzuki et al. (2004a). Agreement among the two hybridization results in the present study (Supplementary Table S2), fixed DNA side and photobiotin-labelled side, supported the reliability of our data. The DNA G + C content of strain JCM $2765^{\mathrm{T}}$ was $48.5 \mathrm{~mol} \%$, which is within the range reported for the genus Lactobacillus (32-53 mol\%) (Kandler \& Weiss, 1986). On the basis of the results presented here, strain JCM $2765^{\mathrm{T}}$ is considered to represent a novel species of the genus Lactobacillus, for which the name Lactobacillus similis sp. nov. is proposed.

\section{Description of Lactobacillus similis sp. nov.}

Lactobacillus similis (si'mi.lis. L. masc. adj. similis similar, resembling, as the type strain is similar to those of $L$. collinoides and $L$. paracollinoides).

Cells cultivated on MRS agar plates are Gram-positive, non-spore-forming, non-motile rods. Cells are $0.5 \mu \mathrm{m}$ in width and variable in length, mostly ranging between 2 and $5 \mu \mathrm{m}$. Colonies are 1-2 $\mathrm{mm}$ in diameter, disc-shaped and white when grown on MRS agar plates at $37{ }^{\circ} \mathrm{C}$ for 2 days. The optimal growth temperature is $37{ }^{\circ} \mathrm{C}$. Grows at $15{ }^{\circ} \mathrm{C}$ but not at $45^{\circ} \mathrm{C}$. Facultatively anaerobic, produces gas from glucose, and produces D- and L-lactic acids heterofermentatively. Catalase is not produced. Aesculin is not

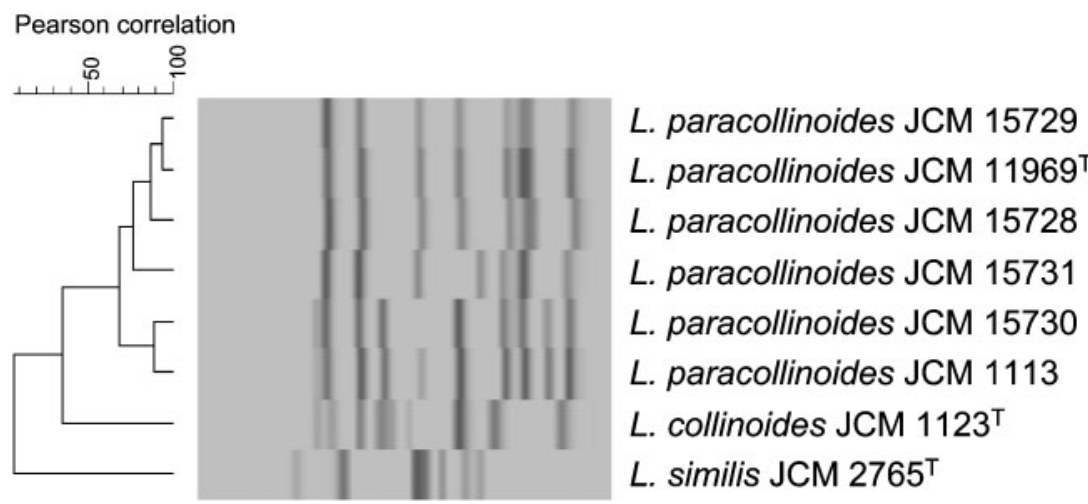

Fig. 2. Dendrogram illustrating the relatedness of the ribotyping patterns of strain JCM $2765^{\top}$, and L. collinoides and L. paracollinoides strains. The dendrogram was analysed via Pearson similarity coefficient analysis and the UPGMA algorithm. 
hydrolysed. In the API $50 \mathrm{CH}$ system, acid is produced from L-arabinose, ribose, $\mathrm{D}$-xylose, methyl $\beta$-D-xyloside, galactose, D-glucose, $\mathrm{N}$-acetylglucosamine, maltose, lactose, melibiose and gluconate. Variable reactions with raffinose and 5-ketogluconate. Acid is not produced from glycerol, erythritol, D-arabinose, L-xylose, adonitol, D-fructose, Dmannose, L-sorbose, rhamnose, dulcitol, inositol, mannitol, sorbitol, methyl $\alpha$-D-mannoside, methyl $\alpha$-D-glucoside, amygdalin, arbutin, aesculin, salicin, cellobiose, sucrose, trehalose, inulin, melezitose, starch, glycogen, xylitol, $\beta$ gentiobiose, turanose, D-lyxose, D-tagatose, D-fucose, Lfucose, D-arabitol or 2-ketogluconate. In the API ZYM system, positive for esterase, esterase lipase, leucine arylamidase, valine arylamidase, phosphatase, naphtholAS-BI-phosphohydrolase, $\alpha$-galactosidase, $\beta$-galactosidase, $\alpha$-glucosidase and $\beta$-glucosidase. Negative reactions for alkaline phosphatase, lipase, cystine arylamidase, trypsin, chymotrypsin, $\beta$-glucuronidase, $N$-acetyl- $\beta$-glucosamidase, $\alpha$-mannosidase and $\alpha$-fucosidase.

The DNA G + C content of strain JCM $2765^{\mathrm{T}}$ is $48.5 \%$. The type strain is JCM $2765^{\mathrm{T}}$ (=LMG $\left.23904^{\mathrm{T}}\right)$, isolated from fermented cane molasses at alcohol plants in Thailand.

\section{Acknowledgements}

We are grateful to Dr Koji Suzuki (Asahi Breweries Ltd) for depositing four reference strains of L. paracollinoides, JCM 1572815731 , with the JCM in addition to JCM $11969^{\mathrm{T}}$. We also thank Ms Natsuko Suzuki for her assistance and Dr J. P. Euzéby for suggestions regarding nomenclature.

\section{References}

Ehrmann, M. A. \& Vogel, R. F. (2005). Taxonomic note "Lactobacillus pastorianus" (Van Laer, 1982) a former synonym for Latobacillus paracollinoides. Syst Appl Microbiol 28, 54-56.

Ezaki, T., Hashimoto, Y. \& Yabuuchi, E. (1989). Fluorometric deoxyribonucleic acid-deoxyribonucleic acid hybridization in microdilution wells as an alternative to membrane filter hybridization in which radioisotopes are used to determine genetic relatedness among bacterial strains. Int J Syst Bacteriol 39, 224-229.

Kandler, O. \& Weiss, N. (1986). Genus Lactobacillus Beijerinck 1901, $212^{\mathrm{AL}}$. In Bergey's Manual of Systematic Bacteriology, vol. 2, pp. 12091234. Edited by P. H. A. Sneath, N. S. Mair, M. E. Sharpe \& J. G. Holts. Baltimore: Williams \& Wilkins.

Kimura, M. (1980). A simple method for estimating evolutionary rates of base substitutions through comparative studies of nucleotide sequences. J Mol Evol 16, 111-120.

Kitahara, M., Takamine, F., Imamura, T. \& Benno, Y. (2001). Clostridium hiranonis sp. nov., a human intestinal bacterium with bile acid $7 \alpha$-dehydroxylating activity. Int J Syst Evol Microbiol 51, 3944.

Saito, H. \& Miura, K. (1963). Preparation of transforming deoxyribonucleic acid by phenol treatment. Biochim Biophys Acta 72, 619-629.

Saitou, N. \& Nei, M. (1987). The neighbor-joining method: a new method for reconstructing phylogenetic trees. Mol Biol Evol 4, 406425.

Seki, M., Kaneuchi, C., Kumnuanta, J., Tantirungij, M., Ohmomo, T. \& Komagata, K. (1989). Identification of lactic acid bacteria isolated from fermented cane molasses at alcohol plants in Thailand. Bull JFCC 5, 80-88.

Suzuki, K., Funahashi, W., Koyanagi, M. \& Yamashita, H. (2004a). Lactobacillus paracollinoides sp. nov., isolated from brewery environments. Int J Syst Evol Microbiol 54, 115-117.

Suzuki, K., Koyanagi, M. \& Yamashita, H. (2004b). Genetic characterization and specific detection of beer-spoilage Lactobacillus sp. LA2 and related strains. J Appl Microbiol 96, 677-683.

Suzuki, K., Ozaki, K. \& Yamashita, H. (2004c). Genetic marker for differentiating beer-spoilage ability of Lactobacillus paracollinoides strains. J Appl Microbiol 97, 712-718.

Suzuki, K., lijima, K., Sakamoto, K., Sami, M. \& Yamashita, H. (2006). A review of hop resistance in beer spoilage lactic acid bacteria. $J$ Inst Brew 112, 173-191.

Tamura, K., Dudley, J., Nei, M. \& Kumar, S. (2007). MEGA4: molecular evolutionary genetics analysis (MEGA) software version 4.0. Mol Biol Evol 24, 1596-1599.

Thompson, J. D., Gibson, T. J., Plewniak, F., Jeanmougin, F. \& Higgins, D. G. (1997). The CLUSTAL_X windows interface: flexible strategies for multiple sequence alignment aided by quality analysis tools. Nucleic Acids Res 25, 4876-4882. 